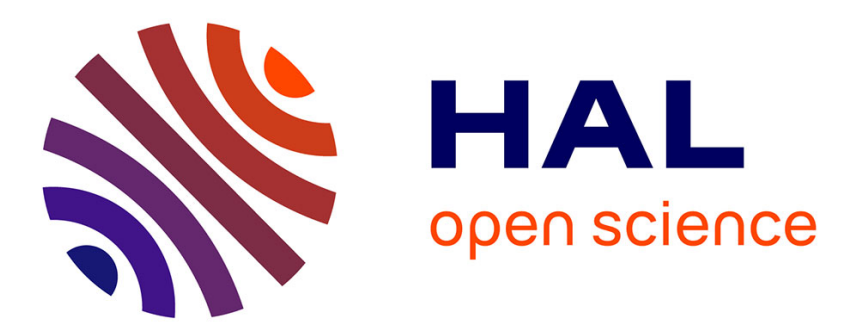

\title{
Structure-based mutagenesis of Penicillium griseofulvum xylanase using computational design.
}

Gwenaëlle André-Leroux, Jean-Guy Berrin, Jacques Georis, Filip Arnaut, Nathalie Juge

\section{- To cite this version:}

Gwenaëlle André-Leroux, Jean-Guy Berrin, Jacques Georis, Filip Arnaut, Nathalie Juge. Structurebased mutagenesis of Penicillium griseofulvum xylanase using computational design.. Proteins - Structure, Function and Bioinformatics, 2008, 72 (4), pp.1298-1307. 10.1002/prot.22029 . hal-02662826

\section{HAL Id: hal-02662826 \\ https://hal.inrae.fr/hal-02662826}

Submitted on 31 May 2020

HAL is a multi-disciplinary open access archive for the deposit and dissemination of scientific research documents, whether they are published or not. The documents may come from teaching and research institutions in France or abroad, or from public or private research centers.
L'archive ouverte pluridisciplinaire HAL, est destinée au dépôt et à la diffusion de documents scientifiques de niveau recherche, publiés ou non, émanant des établissements d'enseignement et de recherche français ou étrangers, des laboratoires publics ou privés. 


\title{
Structure-based mutagenesis of Penicillium griseofulvum xylanase using computational design
}

\author{
Gwénaëlle André-Leroux, ${ }^{1 \dagger}$ Jean-Guy Berrin, ${ }^{2 \dagger}$ Jacques Georis, ${ }^{3}$ \\ Filip Arnaut, ${ }^{4}$ and Nathalie Juge ${ }^{5 \star}$ \\ ${ }^{1}$ Institut Pasteur, Unité de Biochimie Structurale, 25 rue du Dr Roux, 75724 Paris, Cedex XV, France \\ 2 Biosciences ISM2 UMR CNRS 6263, Université Paul Cézanne Aix Marseille III, Av. Escadrille Normandie-Niemen, 13397 Marseille Cedex 20 , France \\ ${ }^{3}$ Puratos Group, rue Bourrie 12, 5300 Andenne, Belgium \\ ${ }^{4}$ Puratos Group, 25 Industrialaan, 1702 Groot-Bijgaarden, Belgium \\ ${ }^{5}$ Institute of Food Research, Colney Lane, Norwich NR4 7UA, United Kingdom
}

\section{ABSTRACT}

Penicillium griseofulvum xylanase (PgXynA) belongs to family 11 glycoside hydrolase. It exhibits unique amino acid features but its three-dimensional structure is not known. Based upon the X-ray structure of Penicillium funiculosum xylanase (PfXynC), we generated a three-dimensional model of PgXynA by homology modeling. The native structure of $P g X y n A$ displayed the overall $\beta$-jelly roll folding common to family 11 xylanases with two large $\beta$-pleated sheets and $a$ single $\alpha$-helix that form a structure resembling a partially closed right hand. Although many features of PgXynA were very similar to previously described enzymes from this family, crucial differences were observed in the loop forming the "thumb" and at the edge of the binding cleft. The robustness of the xylanase was challenged by extensive in silico-based mutagenesis analysis targeting mutations retaining stereochemical and energetical control of the protein folding. On the basis of structural alignments, modeled three-dimensional structure, in silico mutations and docking analysis, we targeted several positions for the replacement of amino acids by site-directed mutagenesis to change substrate and inhibitor specificity, alter $\mathrm{pH}$ profile and improve overall catalytic activity. We demonstrated the crucial role played by Ser44 ${ }_{P g X y n A}$ and Ser129 $9_{P g X n A}$, two residues unique to PgXynA, in conferring distinct specificity to P. griseofulvum xylanase. We showed that the pH optimum of PgXynA could be shifted by -1 to +0.5 units by mutating Ser44 ${ }_{P g X y n A}$ to Asp and Asn, respectively. The S44D and S44N mutants showed only slight alteration in $K_{\mathrm{m}}$ and $V_{\max }$ whereas a S44A mutant lost both $\mathrm{pH}$-dependence profile and activity.
We were able to produce PgXynA S129G mutants with acquired sensitivity to the Xylanase Inhibitor Protein, XIP-I. The replacement of $G \ln 121_{P g X y n A}$, located at the start of the thumb, into an Arg residue resulted in an enzyme that possessed a higher catalytic activity.

Proteins 2008; 72:1298-1307.

(C) 2008 Wiley-Liss, Inc.


side hydrolase family 11; in silico mutagenesis; docking; xylanase inhibitor; site-directed mutagenesis.

\section{INTRODUCTION}

Endo-1,4- $\beta$-xylanases (EC 3.2.1.8 [EC]) catalyze the hydrolysis of the 1,4- $\beta$-xylanosidic linkage in xylan, one of the most abundant plant cell wall polysaccharides in nature. Arabinoxylans (AXs) are often highly complex polysaccharides. ${ }^{1}$ In cereals, the $1,4-\beta$-xylopyranosidic backbone is mainly substituted with monomeric L-arabinofuranosyl and D-glucuronate units by 1,2- and/or 1,3$\alpha$-glycosidic linkages. ${ }^{2}$ Although AXs are minor constituents, they are important for the functionality of cereals in biotechnological processes such as bread-making, gluten-starch separation, animal feed, paper manufacturing, and bioethanol production. ${ }^{3}$ Family 11 xylanases (http:// afmb.cnrs-mrs.fr/CAZY) are highly specific, displaying exclusive substrate specificity toward D-xylose containing

Additional Supporting Information may be found in the online version of this article.

Grant sponsor: European Commission in the Communities 6th Framework Programme (Project HEALTHGRAIN); Grant number: FOOD-CT-2005-514008 ${ }^{\dagger}$ Gwénaëlle André-Leroux and Jean-Guy Berrin equally contributed to the work.

${ }^{*}$ Correspondence to: Nathalie Juge, Institute of Food Research, Colney Lane, Norwich NR4 7UA, UK. E-mail: nathalie.juge@bbsrc.ac.uk or Jacques Georis, Puratos Group, rue Bourrie 12, 5300 Andenne, Belgium. E-mail: jgeoris@beldem.com.

Received 26 October 2007; Revised 23 January 2008; Accepted 1 February 2008

Published online 2 April 2008 in Wiley InterScience (www.interscience.wiley.com). DOI: 10.1002/prot.22029 
substrates and preference for insoluble polymeric substrates, and thus often preferred for technical applications. Therefore, a full understanding of glycoside hydrolase family 11 (GH11) properties is a key step toward the engineering of new xylanases for various applications. The family is highly homologous, ${ }^{4}$ displaying a perfectly conserved jelly-roll framework in which two large $\beta$ pleated sheets and one $\alpha$-helix form a structure that resembles a partly closed right hand. 5,6 The catalytic machinery is composed of two glutamic acid residues, which function as the nucleophile and acid/base catalyst, located in the middle of a cleft formed by the twisted part of the $\beta$ sheet and the $\alpha$ helix. The cleft can accommodate up to seven $\beta-1,4$ xylopyranose units. ${ }^{7}$ Each xylose is accommodated in a subsite which is given a negative or positive number dependent on whether it binds the glycone or aglycone region of the substrate, respectively, with glycosidic bond hydrolysis occurring between the -1 and +1 subsites. ${ }^{8}$ To date, three-dimensional structures of $18 \mathrm{GH} 11$ xylanases are available. Although GH11 enzymes show very closely related three-dimensional structures, xylanases display a wide panel of specificities with regards to $\mathrm{pH}$ activity, thermostability, thermophilicity, inhibition sensitivity. ${ }^{9-17}$ Optimum $\mathrm{pH}$ values are highly variable within this family and range between $\mathrm{pH}$ 2.0 and 4.6 for acidophilic xylanases and between $\mathrm{pH} 5.0$ and $\mathrm{pH} 6.5$ for the so-called "alkaline" xylanases. 18 Structural alignment of GH11 xylanases showed strong correlation between the nature of the residue hydrogen bonded to the general acid/base catalyst and the $\mathrm{pH}$ optimum of these enzymes; it is Asn in "alkaline" xylanases and Asp in those with a more acidic $\mathrm{pH}$ optimum. 4,19 Site-directed mutagenesis studies on acidophilic adaptation have confirmed the importance of residues located in the neighborhood of the glutamic acids, in particular in the thumb and at the edge of the cleft ${ }^{19-21}$

The GH11 xylanase from Penicillium griseofulvum, $\operatorname{PgXynA}$, is unique in that, unlike the xylanases characterized so far, it does not contain the strictly conserved Asp/Asn residue characteristic of the low or high $\mathrm{pH}$ optimum xylanases. PgXynA represents a unique example of a natural xylanase with a Ser residue at this position. We recently enzymatically characterized the enzyme substrate and inhibition specificity. ${ }^{22}$ Like many other carbohydrate-active enzymes, naturally occurring proteinaceous inhibitors of xylanases are present in plant. 17 PgXynA is one of the rare examples of a fungal GH11 xylanase insensitive to the wheat xylanase inhibitor XIP$\mathrm{I}^{22}$. The XIP-I strategy for inhibition of GH11 xylanases involves substrate-mimetic contacts and interactions occluding the active site. ${ }^{23}$ We postulated that structural determinants around the "thumb" region of PgXynA was key to XIP-I resistance, although it remains to be demonstrated. 22,23 These specific characteristics of PgXynA make it an attractive model to study structure-function relationships in the GH11 xylanase family.
Here, we have adopted a rational approach to creating suitably modified xylanase mutants by using computational design methods to guide structure-based sitedirected mutagenesis. The theoretical model, presented in this work, was used as the basis for in silico screening for selecting mutants retaining parental folding. We experimentally evaluated the impact of mutations on novel enzymes with changed substrate and inhibitor specificity, altered $\mathrm{pH}$ profile, and improved overall catalytic activity.

\section{MATERIALS AND METHODS}

\section{Materials}

E. coli DH5 strain was used for DNA manipulation and BL21 strain (DE3) pLys for protein expression (Novagen, Merck Biosciences, Fontenay, France). High purity salt-free oligonucleotides were from Invitrogen (Cergy Pontoise, France), restriction endonucleases and DNA modifying enzymes were from Promega (Madison, WI), pGEX-1 $\lambda \mathrm{T}$ expression vector, GST gluthatione agarose beads, and thrombin protease from Amersham Biosciences (GE healthcare, Orsay, France), BugBuster and benzonase from Novagen, lysosyme, $\beta-\mathrm{D}$ xylose, and dinitrosalicylic acid from Sigma (Lyon, France). Low viscosity wheat arabinoxylan (LVAX) and xylo-oligosaccharides (X2, X3, X4, X5, and X6) were from Megazyme International (Wicklow, Ireland). Purified wheat arabinoxylan substrates with different arabinose to xylose ratio were kindly provided by C. Courtin (Katholieke University Leuven, Belgium).

\section{Bioinformatics}

The signal peptide 24,25 was predicted using the TargetP 1.1 server (http://www.cbs.dtu.dk/services/TargetP/). Sequences were retrieved with Blast ${ }^{26}$ from the ebi website (http://www.ebi.ac.uk/blast/), using PgXynA as the query sequence. ${ }^{27} 2 \mathrm{D}$ alignments were obtained using ClustalW $^{28}$ or T-Coffee ${ }^{29}$ from the ebi (http://www. ebi.ac.uk/Tools/sequence.html) and 3D alignments using ESPRIPT $^{30}$ (http://espript.ibcp.fr/ESPript/ESPript/).

\section{Computational homology modeling}

Homology modeling was performed, using Modeler $6 \mathrm{v} 2,31$ with the B chain of the xylanase of Penicillium funiculosum (PfXynC; 1TE1) 23 as reference sequence and target template. Several models of the PgXynA were generated to satisfy the spatial restraints issued from the alignment with the target protein. The model with the best score function and the correct stereochemistry, as verified by PROCHECK, ${ }^{32}$ was selected and minor repositioning was carried out through short minimization. 33 The superimposition of the target structure and the 


\section{Table I}

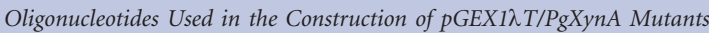

\begin{tabular}{|c|c|}
\hline Name & Oligonucleotide sequence $\left(5^{\prime} \rightarrow 3^{\prime}\right)$ \\
\hline R7Tfor & ATCACCCAGAACGAGACCGGAACCAACGGCGGC \\
\hline R7Trev & GCCGCCGTTGGTTCCGGTCTCGTTCTGGGTG \\
\hline F14Yfor & ACCAACGGCGGCTACTACTACTCTTTTCTGGACC \\
\hline F14Yrev & GGTCCAGAAAGAGTAGTAGTAGCCGCCGTTGG \\
\hline S44Dfor & TGGAAGAATTGCGGAGĀTTTCACCTCTGGCAAGGGC \\
\hline S44Drev &  \\
\hline S44Nfor & TGGAAGAATTGCGGAAATTTCACCTCTGGCAAGGGC \\
\hline S44Nrev &  \\
\hline S44Afor & TGGAAGAATTGCGGAGCTTTCACCTCTGGCAAGGGC \\
\hline S44Arev & 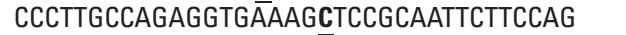 \\
\hline Q121Rfor & ATCTACAAGCATACTCGGGT'̄AACCAGCCTTCG \\
\hline 0121Rrev & CGAAGGCTGGTTGACC̄CGAGTATGCTTGTAG \\
\hline S129Gfor & CAGCCTTCGATCATTGGCGATTCTAGCACCTTCGACCAG \\
\hline S129Grev & CTGGTCGAAGGTGCTAGAATCGCCAATGATCGAAGGCTG \\
\hline$\Delta \mathrm{D} 130 \mathrm{for}$ & CCTTCGATCATTTCGTCTAGCA $\overline{C C T T C G A C C ~}$ \\
\hline$\Delta \mathrm{D} 130$ & GTCGAAGGTGCTAGACGAAATGATCGAAGGC \\
\hline
\end{tabular}

Mutations are in bold underlined.

models was carried out using DaliLite ${ }^{34}$ from the ebi website (http://www.ebi.ac.uk/DaliLite/).

\section{In silico mutation analysis}

In silico mutation analysis was performed to identify the maximum number of mutations that would still guarantee a correct folding of the protein. Since the Nterminal sequence of GH11 xylanases is crucial for the thermostability and thermophylicity of the enzymes, ${ }^{9-14}$ the residues $1-30$ of the PgXynA mature protein were excluded from in silico mutations. Mutational changes were exclusively computed in the variable loop regions to maintain PgXynA topology and activity. The residues were mutated either into their analogues in the sequence of Trichoderma reesei xylanase II (TrXynII; 1XYO) ${ }^{6}$ or into other alternatives issued from Blast to produce a modeled chimera. The stereochemistry of the chimera was verified using PROCHECK and the side chains were optimized through a quick energy minimization. ${ }^{33}$ The potential energy of the final protein was consistent with that of the native model and no steric clash was observed with the mutated residues.

\section{Substrate docking}

A xylohexaose was built by $\beta-1,4$ linking a nondistorted xylopentaose, extracted from the complex with a xylan binding domain of Strepromyces lividans (1MC9), 35 and a xylose moiety added at the reducing end. The xylohexaose was positioned in the catalytic groove of the PgXynA model based upon the positioning of the xylotriose in the active site of the xylanase of Bacillus agaradhaerans $(1 \mathrm{H} 4 \mathrm{H}) .{ }^{36}$ The $(\varphi ; \psi)$ dihedral angles of the sugar chain were slightly revisited to fit into the groove with respect to the $(\varphi ; \psi)$ xylobiose map. ${ }^{37}$ The complex was optimized throughout a cascade of energy minimization and molecular dynamic runs using Charmm forcefield (newly acquired Discovery studio, Accelrys ${ }^{\odot}$ ).

\section{Site-directed mutagenesis, expression, and purification}

Mutations were introduced into the pGEX-1 $\mathrm{T}$ / PgXynA plasmid using the QuickChange ${ }^{\circledR}$ XL sitedirected mutagenesis kit (Stratagene) following manufacturer's recommendations using a pair of overlapping complementary oligonucleotides for each mutation designed to contain the corresponding nucleotide changes. The primers used in this study are given in Table I. E. coli BL21 ( $p L y s$ ) strain was transformed with

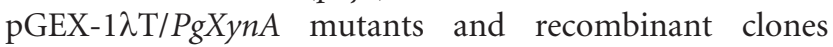
grown at $37^{\circ} \mathrm{C}$ in $\mathrm{LB}$ media containing $50 \mu \mathrm{g} \mathrm{mL}$ ampicillin and $34 \mu \mathrm{g} \mathrm{mL}^{-1}$ chloramphenicol with shaking at $200 \mathrm{rpm}$. Induction of expression, bacterial lysis, purification, and thrombin cleavage of recombinant proteins was performed as previously described. ${ }^{22}$

\section{Protein assays, electrophoresis, and mass spectrometry analysis}

The protein concentration was determined using the Bio-Rad protein assay kit with bovine serum albumin as the standard. SDS-PAGE was performed in $12 \%(\mathrm{w} / \mathrm{v})$ polyacrylamide gel (Bio-Rad, Marnes-la-Coquette, France) using a Pharmacia LMW electrophoresis calibration kit. Native IEF was carried out at $4^{\circ} \mathrm{C}$ in the Bio-Rad gel system, using ampholine carriers of $\mathrm{pH}$ range 3.0-6.0 (Sigma) and pI standards ranging from 4.45 to 8.2 (Bio-Rad). Proteins were visualized either by Coomassie or silver staining. MALDI mass spectra were obtained on an Ettan Maldi-Tof Pro (GE Healthcare Uppsala, 


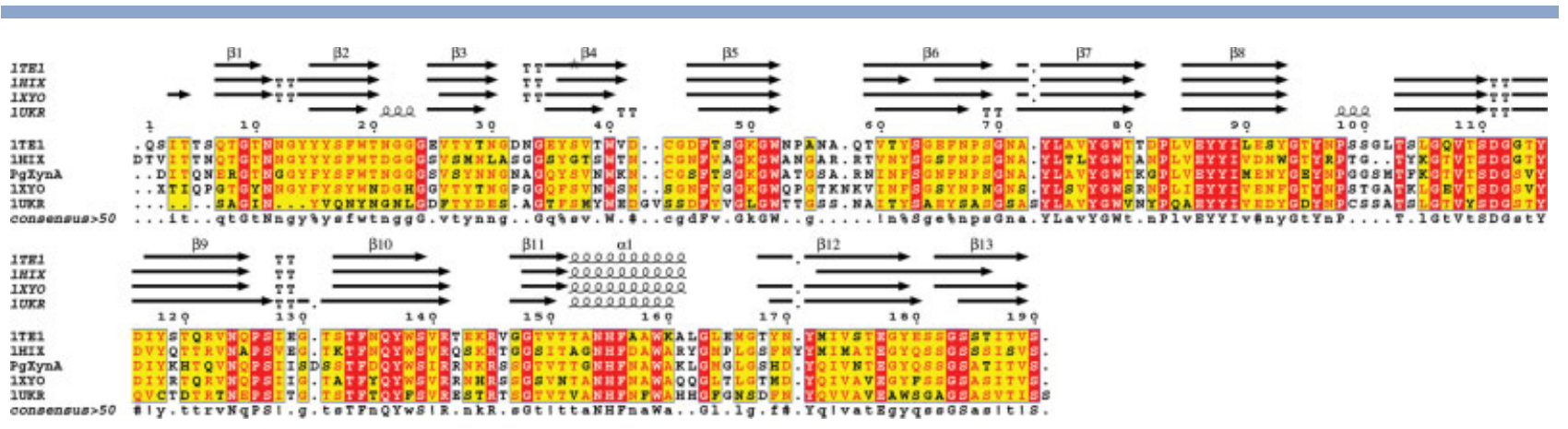

Figure 1

Structural alignment of xylanase sequences from P. griseofulvum (PgXynA), ${ }^{27}$ P. funiculosum (1TE1), ${ }^{23}$ Streptomyces sp. S38 (1HIX), ${ }^{43}$ T. reesei (1XYO) ${ }^{6}$ and A. niger $(1 \mathrm{UKR})^{44}$ using ESPript. The secondary structural elements are shown above for the $3 D$ solved structures. Strictly conserved residues are boxed in red and highly conserved ones colored in red.

Sweden) operating in positive linear mode with delayed extraction. The samples were treated as previously described. 22

\section{Xylanase and inhibition activity assays}

Purified xylanase activity was measured as described previously. ${ }^{38}$ Briefly, $20 \mu \mathrm{L}$ of enzyme (PgXynA wt or mutants) was mixed with $180 \mu \mathrm{L}$ of substrate in McIlvaine's buffer $\mathrm{pH} 5.5$ at $30^{\circ} \mathrm{C}$ for $5 \mathrm{~min}$. The reaction was terminated by the addition of $300 \mu \mathrm{L}$ dinitrosalicylic acid (DNS) reagent and boiled for $5 \mathrm{~min}$. The reactions were cooled and centrifuged for $5 \mathrm{~min}$ at $13,000 \mathrm{rpm}$ and $200 \mu \mathrm{L}$ was transferred to a microtitre plate. The absorbance at $550 \mathrm{~nm}$ was measured relative to a xylose standard curve $\left(0-180 \mu \mathrm{g} \mathrm{mL}^{-1}\right)$. One unit of xylanase activity was defined as the amount of protein that released $1 \mu \mathrm{mol}$ of xylose $/ \mathrm{min}$ at $30^{\circ} \mathrm{C}$ and $\mathrm{pH} 5.5$. Optimal $\mathrm{pH}$ for xylanase activity was estimated using LVAX (10 $\mathrm{mg} \mathrm{mL}^{-1}$ ) in McIlvaine's buffer in a $\mathrm{pH}$ range of 2.4-7.5. For determination of apparent Michaelis-Menten constants, the initial velocities of the enzymes were measured at $30^{\circ} \mathrm{C}$ in McIlvaine's buffer, $\mathrm{pH}$ 5.5, with substrate concentrations ranging from 2 to $20 \mathrm{mg} \mathrm{mL}^{-1}$. Because of the heterogeneous nature of polymeric substrates, their molar concentrations could not be calculated. Consequently, only an apparent value for the Michaelis constant, $K_{\mathrm{m}(\mathrm{app})}$, was determined. The kinetic parameters were estimated using weighted nonlinear squares regression analysis with the Grafit software (Biosoft, Cambridge, UK). Inhibition was detected by adding increasing molar equivalents of XIP-I ${ }^{38}$ to the enzyme solution up to a maximum molar ratio of $30: 1$. The $K i$ value was determined at different concentrations of LVAX substrate $(2-18 \mathrm{mg} / \mathrm{mL})$ at $30^{\circ} \mathrm{C}$ and $\mathrm{pH} 5.5$ in the presence of various amounts of XIP-I.

\section{High performance anion exchange chromatography-pulsed amperometric detection}

Products generated by hydrolysis of wheat arabinoxylans by wild type and mutated xylanases were analyzed by HPAEC (Dionex, Sunnyvale, CA) equipped with a Carbo-Pac PA-100 analytical column $(250 \times 4 \mathrm{~mm})$, a GP40 gradient pump and an AS3500 auto sampler. The hydrolysis was carried out using appropriate enzyme and substrate concentrations in McIlvaine's buffer $\mathrm{pH}$ 5.5. The enzymatic reaction was stopped by the addition of $1 M$ sodium potassium tartrate $\mathrm{pH} 11.5$ and boiled for 5 min prior to injection $(20 \mu \mathrm{L})$ on the HPAEC system. Elution was carried out as previously described. ${ }^{22}$ The effluent was monitored using an ED40 electrochemical detector.

\section{RESULTS}

\section{Model of PgXynA and substrate docking}

Since no crystal structure was available, a molecular model of PgXynA was generated using the P. funiculosum xylanase (PfXynC; 1TE1)23 as the template (Fig. 1). As expected, PgXynA exhibited the overall $\beta$-jelly-roll shape, typical of GH11 xylanases and reminiscent of a right hand, with the palm located between Thr152 $2_{\mathrm{PgXynA}}$ and Leu162 $2_{\operatorname{PgXyn}}$, the cord by a loop positioned between Gly93 $3_{\text {PgXnA }}$ and Lys $104_{\text {PgXynA }}$ and the thumb by a hairpin loop located between Val122 $2_{\mathrm{PgXynA}}$ and Ser131 $1_{\mathrm{PgXynA}}$. By analogy with other GH11 xylanases, $5,6,23,39,40$ the cleft is paved with strictly conserved aromatic residues that line up the active site with putative $\pi$ stacking interactions. ${ }^{41}$ The carbonyl oxygens of the predicted catalytic residues, Glu85 $5_{\operatorname{Pg} X n A}$ (catalytic nucleophile) and Glu177 ${ }_{\text {PgXynA }}$ (catalytic acid-base), are located less than $6 \AA$ apart in the model, which is consistent with the cata- 


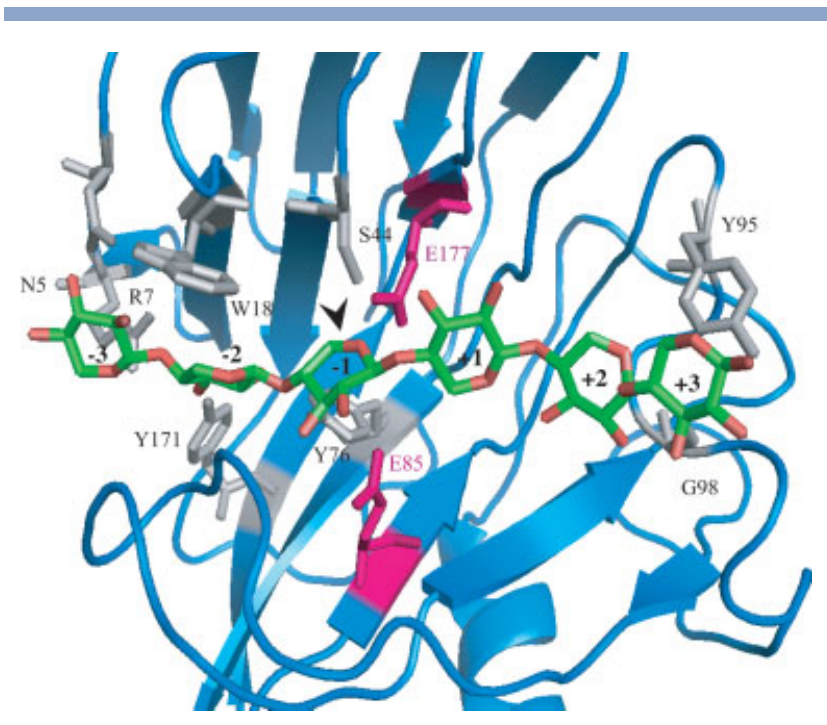

Figure 2

Close up view of the active site of PgXynA model structure, with the bound modeled xylohexaose. The residues predicted to participate in substrate binding through hydrogen bonding or van der Waals interactions are labeled. The xylohexaose molecule, modeled in the energetically most favorable conformation, is atom-sticked and atom-type colored in green. The distortion from ${ }^{4} C_{1}$ to ${ }^{2} B_{5}$ for the xylose moiety bound to subsite $(-1)$ is indicated by an arrow.

lytic apparatus of a "retaining" glycoside hydrolase. ${ }^{39,40,42}$ The nucleophile belongs to a highly conserved cluster [V, I]-E-Y-Y [I, V], whereas the acid/base belongs to the less conserved [T, V]-E-G-Y motif.

The superimposition of the PgXynA model with that of the PfXynC 3D structure led to a root mean square deviations of $0.2 \AA$ between the $\mathrm{C} \alpha$ of 189 residues and a $Z$ score of 38.1. The significant structural differences between PgXynA and PfXynC are mostly located between

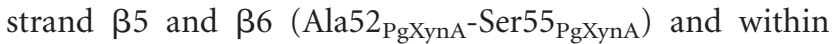
the thumb region (Ile127 $7_{\text {PgXynA }}-$ Ser $131_{\text {PgXynA }}$ ) that includes the amino acid insertion of Asp $130_{\operatorname{PgXynA}}$. Other differences concern amino acid side chains spread along the sequence defining the catalytic groove: $\operatorname{Arg} 7_{\mathrm{PgXynA}}$ versus Thr7 $7_{\text {PfXynC, }}$ Phe1 $4_{\text {PgXynA }}$ versus Tyr14 $4_{\text {PfXynC, }}$ Ser44 $_{\text {PgXynA }}$ versus Asp44 $4_{\text {PfXynC, Ala5 }} 2_{\text {PgXynA }}$ versus Asn52 PfXynC, Met89 $_{\text {PgXynA }}$ versus Leu89 ${ }_{\text {PfXynC, Gly98 }}$ PgXynA versus Ser98 ${ }_{\text {PfXynC, Gly99 }}{ }_{\text {PgXynA }}$ versus Ser99 ${ }_{\text {PfXynC, }}$ Met101 $1_{\text {PgXnA }}$ versus Leu101 PfXynC, His119 $_{\text {PgXynA }}$ versus


Ser129 ${ }_{\text {PgXynA }}$ versus Gly129 PfXynC $_{\text {(Fig. 1). These residues }}$ differed in the length, charge and positioning of their $3 \mathrm{D}$ side-chains and were also distinct in other GH11 xylanases for example, xylanases from Streptomyces sp. S38 (1HIX), ${ }^{43}$ T. reesei (TrXynII; $\left.1 \mathrm{XYO}\right)^{6}$ and Aspergillus niger (1UKR) 44 (Fig. 1). Among those, $\operatorname{Arg} 7_{\mathrm{PgXynA}}$, Phe14 $4_{\text {PgXynA }}$, Ser44 $4_{\text {ggXynA }}$, Gln121 $1_{\text {PgXynA }}$, and Asp130 ${ }_{\text {PgXynA }}$ were targeted for experimental site-directed mutations (discussed later).
The 3D homology model was further challenged in silico to determine the robustness of the xylanase against mutations. A sequence variation of up to $16 \%$ was permitted to produce a xylanase retaining stereochemical and energetical control of protein folding. The permissive mutations are listed in "Supplementary Materials" and a xylanase chimera (not shown) was homology modeled based on TrXynII (XYO) ${ }^{6}$ that displayed most of the identified mutations (see MATERIAL AND METHODS).

To aid in the design of active site mutants, a modeled xylohexaose was docked in the active site of the PgXynA model, from subsites $(-3)$ to $(+3)$ (Fig. 2). The docking of the substrate revealed similar interactions with residues that contact the sugar rings in the experimentally solved structure of GH11 xylanases in complex with oligosaccharides and covalent inhibitors. 5,6,23,36,39-44 From these studies, it can be suggested that Asn $5_{\operatorname{PgXynA}}$, $\operatorname{Arg} 7_{\operatorname{PgXynA}}$, and $\operatorname{Trp} 18_{\operatorname{PgXynA}}$ define the subsite $(-3)$; Trp $18_{\operatorname{PgXynA}}$ stacks the xylose in subsite $(-2)$, assisted by hydrogen bonding of $\operatorname{Arg} 7_{\operatorname{PgXynA}}, \operatorname{Tyr} 76_{\operatorname{PgXynA}}$, and Tyr171 $1_{\text {PgXynA }}$; Ser44 $4_{\text {PgXynA }}$, Pro125 $5_{\text {PgXynA }}$, and Glu177 ${ }_{\text {PgXynA }}$ define subsite $(-1)$. Ser4 $4_{\text {PgXynA }}$ and Tyr72 ${ }_{\mathrm{PgXynA}}$ are engaged in hydrogen bonding of xylose in subsite $(+1)$. The xylose in subsite $(+2)$ appears hydrogen bonded to the carbonyl oxygen of Gly $98_{\operatorname{PgXynA}}$ and the reducing ring in subsite $(+3) \pi$-stacked to $\operatorname{Tyr} 95_{\operatorname{PgX} y n A}$. The PgXynA model is in accordance with previous kinetic analyses reporting the predominant production of xylotriose from xylohexaose, suggesting that the specificity region of the xylanase spans about six xylose units. ${ }^{22}$ After several runs of molecular dynamic with no constraints, the xylose moiety located at subsite $(-1)$ underwent a distortion from the ${ }^{4} \mathrm{C}_{1}$ chair conformation towards the boat ${ }^{2} \mathrm{~B}_{5}$ geometry, as assessed by the measurements of the Pucker parameters. ${ }^{45}$ This conformation allowed atoms C5, O5, C1, and C2 of the sugar in subsite $(-1)$ to achieve coplanarity (Fig. 2). This is suggestive of a transition state conformation since a ${ }^{2} \mathrm{~B}_{5}$ geometry was observed in the crystal structure of a xylotriose bound to subsite $(-1)$ of $B$. agaradhaerens xylanase ${ }^{36}$ where it is essential to the formation of an oxocarbenium ion-like transition state, characteristic of the double-displacement catalytic mechanism. This distortion was also observed in the crystal structure of a 2-fluoro-xylose residue bound to subsite $(-1)$ of $B$. circulans xylanase. ${ }^{39}$ To summarize, the docking of a substrate analogue into the catalytic site of PgXynA highlighted Arg7 ${ }_{P_{g X y n A}}$ and Ser4 $4_{P_{g X y n A}}$ as relevant target for modifying substrate binding in GH11 xylanases.

\section{Site-directed mutagenesis}

Based upon sequence alignments and analysis of the PgXynA 3D model, mutations R7T, F14Y, S44A, S44D, S44N, Q121R, S129G, $\Delta$ D130 were introduced by sitedirected mutagenesis and some combined in double 
Table II

Specific Activities and Kinetic Parameters of wt and Mutant PgXynA Xylanases on Wheat Arabinoxylan (LVAX)

\begin{tabular}{|c|c|c|c|c|c|}
\hline PgXynA & $S A^{a}\left(U_{m g}^{-1}\right)$ & $K_{\mathrm{m}(\mathrm{app})}\left(\mathrm{mg} \mathrm{mL}^{-1}\right)$ & $k_{\text {cat }}\left(\mathrm{s}^{-1}\right)$ & $k_{\text {cat }} / K_{\mathrm{m}(\mathrm{app})}\left(\mathrm{mL} \mathrm{s}^{-1} \mathrm{mg}^{-1}\right)$ & $K_{\mathrm{i}}(\mathrm{n} M)$ \\
\hline$\overline{w t}$ & $1100 \pm 84$ & $3.8 \pm 0.7$ & $545 \pm 29$ & 143 & $\mathrm{~N} / \mathrm{A}$ \\
\hline R7T & $1085 \pm 71$ & $11.9 \pm 1.5$ & $448 \pm 25$ & 38 & N/A \\
\hline F14Y & $1226 \pm 92$ & $5.9 \pm 0.8$ & $773 \pm 47$ & 131 & N/A \\
\hline S44D & $493 \pm 31$ & $4.4 \pm 0.7$ & $298 \pm 17$ & 66 & N/A \\
\hline S44N & $954 \pm 42$ & $3.9 \pm 0.4$ & $454 \pm 24$ & 116 & N/A \\
\hline S44A & $45 \pm 3$ & $4.6 \pm 0.8$ & $27 \pm 2$ & 6 & $\mathrm{~N} / \mathrm{A}$ \\
\hline Q121R & $1464 \pm 46$ & $7.7 \pm 1.5$ & $1063 \pm 86$ & 138 & $\mathrm{~N} / \mathrm{A}$ \\
\hline S129G & $764 \pm 31$ & $7.5 \pm 0.9$ & $484 \pm 28$ & 64 & 82 \\
\hline$S 129 G / \Delta D 130$ & $627 \pm 40$ & $6.4 \pm 0.3$ & $457 \pm 8$ & 71 & 3.9 \\
\hline$\Delta \mathrm{D} 130$ & $1146 \pm 34$ & $5.7 \pm 1.0$ & $601 \pm 42$ & 105 & N/A \\
\hline S129G/S44D & $95 \pm 4$ & ND & ND & ND & ND \\
\hline S129G/S44N & $866 \pm 22$ & $6.5 \pm 1.0$ & $543 \pm 45$ & 83 & 90 \\
\hline
\end{tabular}

N/A, not applicable; ND, not determined.

${ }^{\text {a }}$ Specific Activity at $30^{\circ} \mathrm{C}$, pH 5.5 in Mcllvaine's buffer, substrate concentration $10 \mathrm{mg} \mathrm{ml}^{-1}$.

mutants. All mutant and wild-type (wt) xylanases were expressed in E. coli, purified as previously described and characterized in terms of molecular mass using mass spectrometry, $\mathrm{pI}$ and activity. The specific activity and kinetics parameters $\left(k_{\text {cat }}, K_{\mathrm{m}(\mathrm{app})}, K_{\mathrm{i}}\right)$ of PgXynA xylanases were determined using wheat arabinoxylan (LVAX) as substrate (Table II).

\section{Active site mutants}

The R7T and F14Y mutants retained specific activity comparable to the wt-PgXynA (Table II). The activity of

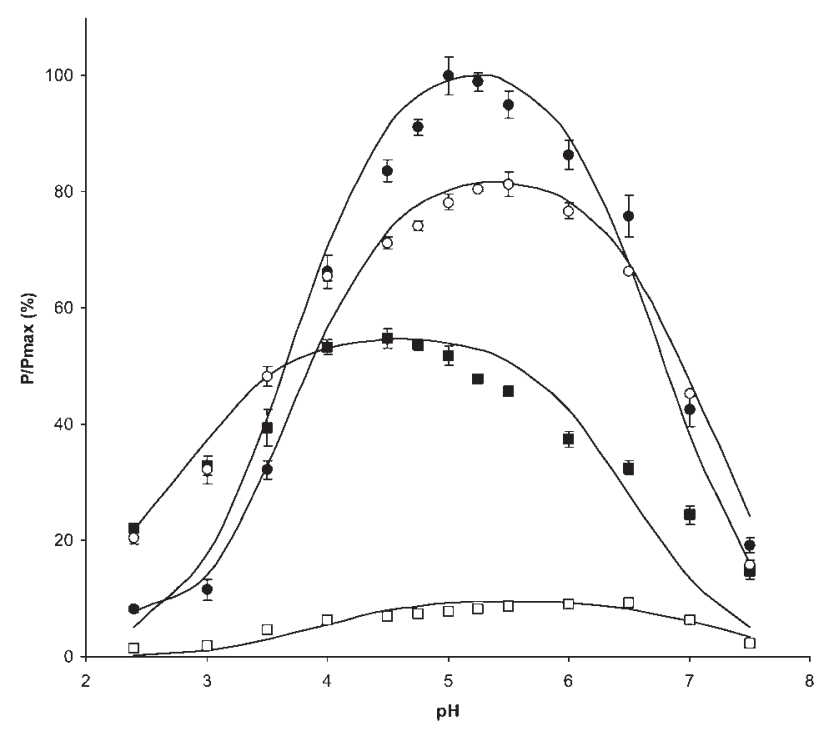

Figure 3

$p H$ dependence of the wild-type $(\bigcirc)$ and mutant enzymes S44N $(O), S 44 D$ $(\square)$, and S44A ( $\square$ ). The experimental data were fitted (solid lines) using the equation as described in de Lemos Esteves and coll. ${ }^{21} \mathrm{P}$ represents the activity measured at various $\mathrm{pH}$, and $\mathrm{P}_{\max }$ the maximum activity observed for wild-typ PgXynA at pH 5.0. The following pKa values were used to fit the equation: $w t$, 3.7 and 6.7; S44D, 2.5 and 6.5; S44N, 3.5 and 7.2; S44A, 3.9 and 7.2. the wild-type and Ser44 mutants was investigated at $\mathrm{pH}$ values between 2.4 and 7.5, using LVAX as substrate. The wt-PgXynA ( $\mathrm{pI}$ 8.6), as also reported for PfXynC (pI 4.2 ), displayed over $50 \%$ activity in the $\mathrm{pH}$ range $4.5-6.5$ with an optimum $\mathrm{pH}$ near 5.0 (Fig. 3). However, around $30 \%$ of activity was still observed at $\mathrm{pH} 7.3$ for PgXynA whereas no activity was detected at this $\mathrm{pH}$ for PfXynC. ${ }^{22}$ Furthermore PgXynA retained full activity for $2 \mathrm{~h}$ in the $\mathrm{pH}$ range $4.0-7.5$ at $30^{\circ} \mathrm{C}$ (not shown). In addition and in contrast to all GH11 "alkaline" xylanases characterized so far, PgXynA contains a Ser residue instead of the expected Asn residue adjacent to the acidbase catalyst (Fig. 2). This unique specificity of the P. griseofulvum xylanase A was investigated further by sitedirected mutagenesis. The S44A mutation abolished the bell-shaped $\mathrm{pH}$-activity profile of PgXynA whereas mutations to Asp or Asn resulted in a shift in the $\mathrm{pH}$ profile by $\sim 0.5-1.0 \mathrm{pH}$ units (Fig. 3). The S44D mutation shifted the activity to acidic $\mathrm{pHs}$ by $\sim 1$ unit, decreasing the optimum $\mathrm{pH}$ of PgXynA to 4.5 whereas the S44N mutation had the opposite effect, shifting the activity to alkaline $\mathrm{pHs}$ by $\sim 0.5$ with a $\mathrm{pH}$ optimum of 5.5. Moreover the S44D mutant had a broader $\mathrm{pH}$ profile retaining $\sim 60 \%$ of its maximum activity at $\mathrm{pH} 3.0$, where the wild-type enzyme is almost inactive (Fig. 3). This is consistent with previous mutagenesis studies of GH11 xylanases demonstrating correlation between the presence of an Asn or an Asp residue at position 44 (PgXynA numbering) and the $\mathrm{pH}$ optimum of the mutated enzymes. The substitutions led to an overall reduction in activity (S44N> S44D $>$ S44A) mainly due to a reduction in $k_{\text {cat }}$ whereas the apparent affinity remained unchanged (Table II).

\section{"Thumb" mutants}

Among the "thumb" mutants, the Q121R mutant showed a marked $33 \%$ increase in specific activity, mainly due to a two-fold increase in catalytic turnover $\left(k_{\text {cat }}\right)$ (Table II). To evaluate the mode of action of the 

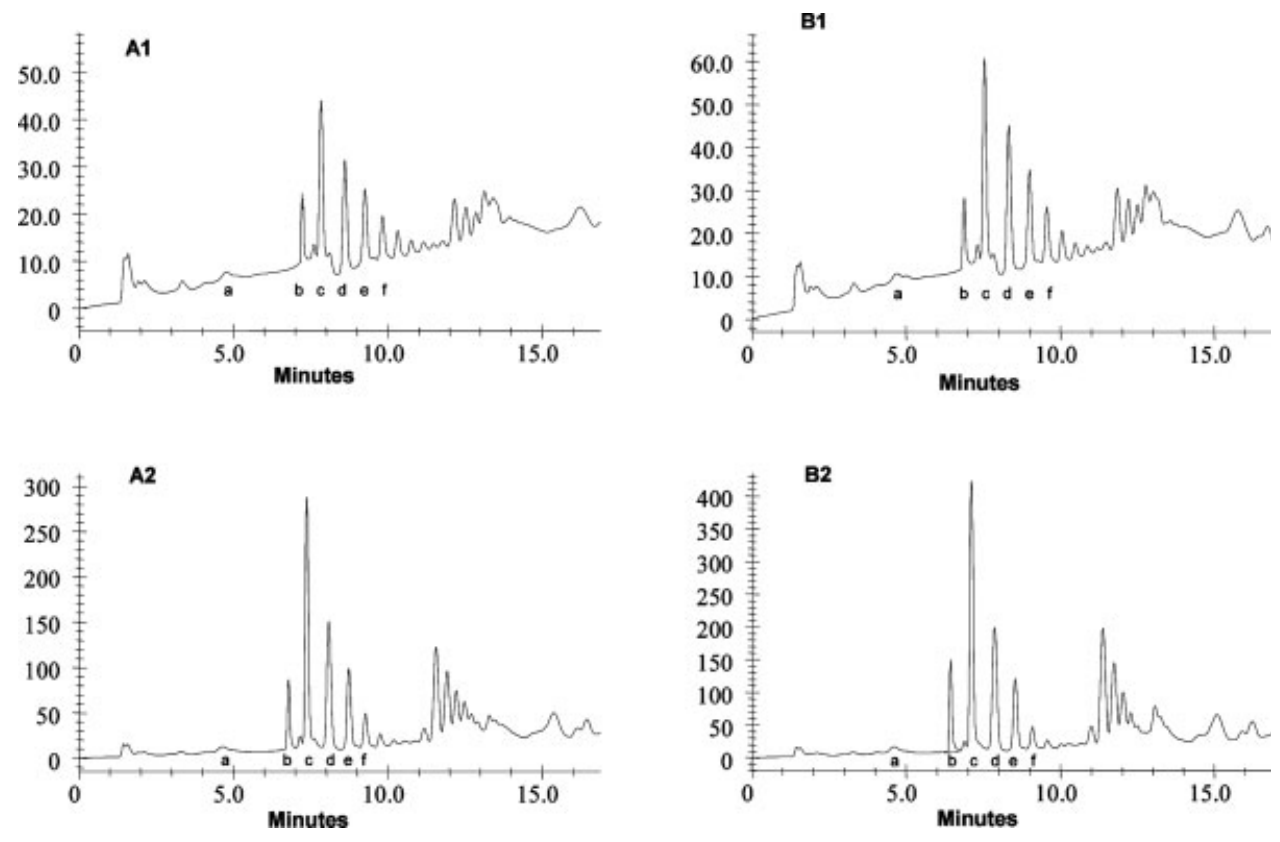

Figure 4

HPAEC analysis of wheat LVAX hydrolysis by PgXynA wt (A) and Q121R (B). PgXynA wt and mutant (20 nM) were incubated with $5 \mathrm{mg} / \mathrm{mL}$ wheat arabinoxylan in McIlvaine's buffer of pH 5.5 at $30^{\circ} \mathrm{C}$. The reaction was stopped after $10 \mathrm{~min}$ (1) and $60 \mathrm{~min}(2)$, and subjected to HPAEC analysis: $a, b, c, d$, $e$, and $f$ correspond to xylose, xylobiose, xylotriose, xylotetraose, xylopentaose, and xylohexaose, respectively.

mutant, the hydrolysis products generated on highly polymeric substrate were analyzed by high-performance anion exchange chromatography (HPAEC-PAD). The Q121R mutation did not alter the hydrolysis product profile of wheat arabinoxylan, that is, a mixture of xylooligosaccharides was generated during the initial stages of hydrolysis and as the reaction continued, the oligosaccharides were progressively degraded yielding principally xylobiose and xylotriose towards the end of the reaction, but an overall increase of released xylo-oligosaccharides products was observed (Fig. 4). The Q121R mutant was further tested on wheat arabinoxylan fractions of different arabinose/xylose ratio in order to test the effect of the substitution on the specific activity. The mutated enzyme showed highest increase in activity on low substituted xylan: A/X $0.36>\mathrm{A} / \mathrm{X} 0.49>\mathrm{A} / \mathrm{X} 0.68$, with a maximum increase of up to $50 \%$ on $\mathrm{A} / \mathrm{X} 0.36$ compared to the wild-type (not shown). This is consistent with the generally accepted statement that GH11 xylanases preferentially cleave in unsubstituted regions of the AX backbone, whereas GH10 cleave in decorated regions, being less hampered by the presence of D-glucuronate and L-arabinofuranosyl substituents along the xylan backbone. 2,42

The wheat protein inhibitor XIP-I inhibits fungal but not bacterial GH11 xylanases. The structural basis for the inhibition came from the detailed analysis of the 3D structure of XIP-I in complex with PfXynC. ${ }^{23}$ Inhibition of the GH11 xylanase is mediated by the insertion of a

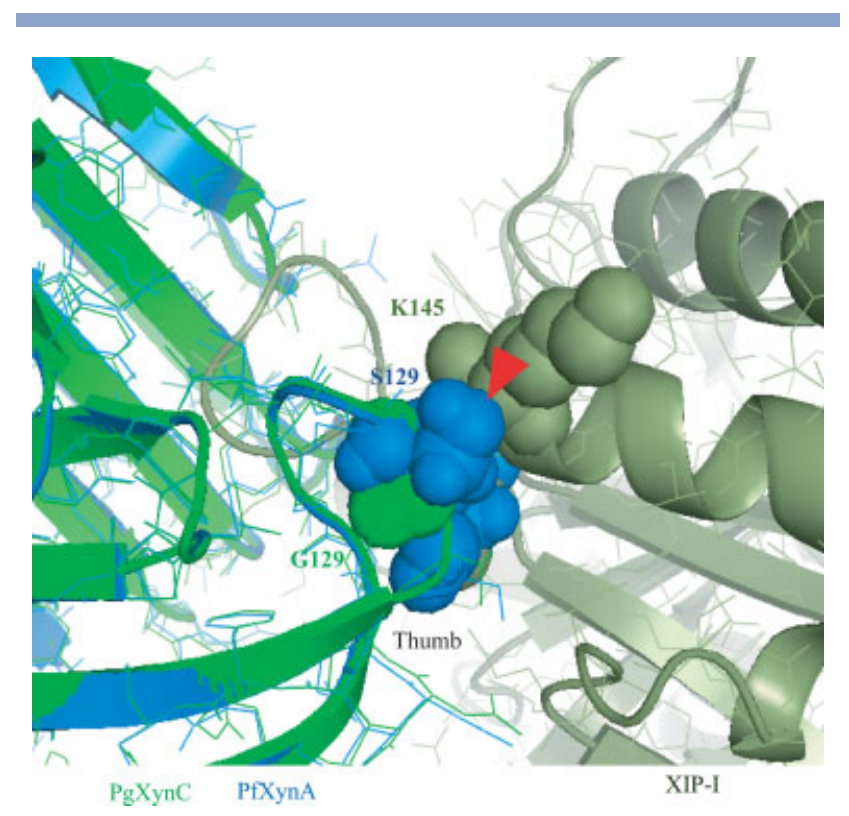

Figure 5

Evidence of steric hindrance between PgXynA and XIP-I. The inhibitor was extracted from its solved complex with PfXynC (1TE1). ${ }^{23}$ The backbone of $P g X y n A$ was superimposed onto the coordinates of the PfXynC backbone, bound to XIP-I. PfXynC and PgXynA are in green and blue, respectively whereas XIP-I is represented in light green. The Gly129 $9_{P f X y n C}$, Ser $129_{P g X y n A}$ and Lys $145_{X I P-I}$ side-chains are in CPK spheres. The clash between Ser129PgXynA and Lys145 XIP-I is indicated by an arrow. 
XIP-I П-shaped loop (148-153) into the enzyme active site but interactions with regions (thumb and palm) surrounding the entrance of the active site groove are also important recognition determinants for XIP-I, with the overall shape and amino acid composition of the thumb playing a significant role in inhibitor binding. As demonstrated earlier, the wt-PgXynA is insensitive to XIP- $\mathrm{I}^{22}$. In the present work, we showed that deletion of Asp $130_{\operatorname{PgXynA}}$, protruding from the thumb region, did not alter inhibition sensitivity of the mutated enzyme whereas the S129G and the S129G/S44N substitutions conferred inhibition sensitivity with $K_{\mathrm{i}}=82 \mathrm{n} M$ and $K_{\mathrm{i}}=90 \mathrm{nM}$, respectively (Table II and Fig. 5). The low specific activity of the S129G/S44D mutant prevented to study the effect of inhibition by XIP-I. However, a variant highly sensitive to the inhibitor was obtained when combining deletion of Asp130 with S129G mutation. The S129G/ $/$ D130 mutant had a $K_{\mathrm{i}}=3.9 \mathrm{nM}$, which is one of the lowest compared to tested natural enzymes. ${ }^{16}$ These results demonstrate that although deletion of Asp $130_{\operatorname{PgXynA}}$ is required to reach optimum inhibition level, the Asp130 insertion is not per se responsible for the lack of interaction with XIP-I and that Gly129 $9_{\operatorname{PgXynA}}$ plays a determinant role in the interaction with the inhibitor by ensuring correct positioning of the loop and preventing steric hindrance.

\section{DIscussion}

An enzyme in general is known for its specificity due to extreme sensitivity at crucial positions. Nevertheless, to maintain its folding and more importantly its function, an enzyme has to be robust to mutational events. Homology modeling with the target templates of PfXynC and TrXynII computed a native and a chimera model of PgXynA protein, respectively. The permissive level of in silico mutations reached $16 \%$, confirming the predicted robustness of the GH11 xylanase folding. ${ }^{4,5}$

In addition, bioinformatics, docking, and molecular dynamics were used to identify residues crucial for substrate binding, $\mathrm{pH}$ activity or motion flexibility. These predictions were validated through experimental site-directed mutagenesis. In particular, two residues highly conserved in other GH11 xylanases but different in PgXynA have been shown to be functionally significant. These are Ser44 and Ser129 (Fig. 1). Ser44 belongs to the highly conserved motif [GN/DFV/LxGKGW] where it replaces the conserved Asp or Asn residue found in "acidic" and "alkaline" GH11 xylanases, respectively. 4,18-21,44,46 These neighboring residues of the acid/base catalyst determine the $\mathrm{pH}$ activity range of the enzyme following a "reverse protonation" mechanism dependent upon the electrostatic linkage of the groups involved in the interaction. ${ }^{44}$ In the present study, we showed that we could drive the optimum $\mathrm{pH}$ activity of PgXynA toward the $\mathrm{pH}$ of the acidic or alkaline xylanases by mutating the Ser residue into an Asp or Asn residue, respectively. However these mutations occurred at the expense of the activity since these groups may be involved in ground and/or transition state binding. In the PgXynA model structure with the modeled xylohexaose, the hydroxyl group of the Ser residue appears to be involved in bidentate hydrogen bonding of xylose residues in subsite $(-1)$ and $(+1)$, suggesting that it could participate in the distortion of the nonreducing xylose in $(-1)$ towards its transition state geometry, thus leading to a boat geometry. Regarding the $\mathrm{pH}$ dependency of the enzymes, the side chain of the serine is significantly shorter than an aspartic acid or an asparagine residue so its participation in the protonation state of the neighboring catalytic Glu177 is jeopardized. The increased efficiency of the S44D mutant under more acidic conditions could be attributed to the formation of a shorter hydrogen bond between Asp44 side-chain and the acid/base catalyst Glu177 (Fig. 2). S44N has the same side-chain length as compared to its aspartate counterpart but does not contain any charge and thus cannot hydrogen bond Glu177. These results complement previous mutational studies where the Asp/Asn residues were substituted by their counterparts in other GH11 xylanases. ${ }^{4,18-21,44,46}$ Particularly relevant to the present work was the mutation of Asp to Ser in the acidic xylanase C from Aspergillus kawachii, leading to a shift in $\mathrm{pH}$ optimum from 2 to 4 , thus slightly lower than the mutation to Asn which shifted the $\mathrm{pH}$ optimum of the mutant to $5 .^{20}$

The "thumb" region is known to play an important role in the interaction of GH11 xylanases with XIP-I, as first demonstrated by site-directed mutagenesis of xylanase from A. niger 47 and later on xylanases from Fusarium graminearum 48 and Bacillus subtilis. ${ }^{49}$ In the present work, analysis of S129G, $\Delta \mathrm{D} 130, \mathrm{~S} 129 \mathrm{G} / \mathrm{S} 44 \mathrm{~N}$, and S129G/ $\Delta$ D130 mutants showed that the insertion of Asp130 within the thumb region of PgXynA was not solely responsible for the lack of inhibition of the enzyme by XIP$\mathrm{I}$, as previously suggested. ${ }^{22}$ Deleting Asp $130_{\mathrm{PgXynA}}$ and substituting the adjacent Ser129 $\operatorname{PgXynA}$ by Gly, as it is in PfXynC, restored inhibition of PgXynA by XIP-I. In the model of PgXynA, the Asp130 ${ }_{\mathrm{PgX} X \mathrm{~A}}$ and Ser129 ${ }_{\mathrm{PgX} \text { XnA }}$ side chains appear to clash against Asn147 XIP-I and Lys145 XIP-I, respectively, hampering interaction in that area (Fig. 5). Together, these results showed that the resistance of PgXynA to XIP-I is mostly due to the lack of flexibility of the loop, causing steric clashes between PgXynA and the inhibitor. A recent computational study of the thumb loop flexibility of a xylanase from Thermobacillus xylaniliticus using geometric path analysis indicated a motion amplitude of several Angströms. ${ }^{50}$ High level of inhibition requires the loop to adopt an optimum conformation governed by the nature of the 129-130 residues in PgXynA.

Finally, we have improved the overall catalytic efficiency of some of the PgXynA mutants by mutating residues outside the core of the active site to avoid perturbing the exquisitely balanced network of complex interac- 
tions that surround the catalytic groups. In particular the Q121R variant, located at the beginning of the "thumb", showed higher specific activity on wheat arabinoxylan and retained product hydrolysis profile similar to the wild-type. Although Gln $121_{\mathrm{PgXynA}}$ is not seen to be involved in any interaction in the PgXynA model, substitution into an arginine leads to an extensive hydrogen bonding network with Gln124, Gln136, and the catalytic residue Glu85 (not shown). In addition, Arg121 appears to hydrogen bond the $\mathrm{O}_{2}$ of xylose in subsite $(-1)$ and $\mathrm{O}_{4}$ and $\mathrm{O}_{5}$ in subsite $(+1)$, which could explain the enhanced affinity toward the substrate through stabilization of the ground state during catalysis (not shown).

\section{CONCLUSIONS}

In summary, we have devised a computational procedure to identify residues to be mutated with no change in the stereochemical and energetical control of the protein folding. Other mutations were suggested to modify substrate binding, $\mathrm{pH}$ activity or selective inhibition and experimental analysis confirmed that the procedure was successful in identifying xylanase mutants with novel specificity. This combination is a straightforward alternative to directed evolution and high throughput screening. ${ }^{51}$ By using molecular modeling, docking, in silico analysis and site-directed mutagenesis, we have generated knowledge to improve family 11 xylanases.

\section{ACKNOWLEDGMENTS}

This publication reflects only author's views and the Community is not liable for any use that may be made of the information contained in this publication. We thank Claude Villard for mass spectrometry analysis and Christophe Courtin for providing purified wheat arabinoxylan substrates with different arabinose to xylose ratio.

\section{REFERENCES}

1. Fincher GB. Molecular and cellular biology associated with endosperm mobilization in germinating cereal grains. Annu Rev Plant Physiol Plant Mol Biol 1989;40:305-346.

2. Saulnier L, Sado PE, Branlard G, Charmet G, Guillon F. Wheat arabinoxylans: exploiting variation in amount and composition to develop enhanced varieties. J Cereal Sci 2007;46: 261-281.

3. Polizeli ML, Rizzatti AC, Monti R, Terenzi HF, Jorge JA, Amorim DS. Xylanases from fungi: properties and industrial applications. Appl Microbiol Biotechnol 2005;67:577-591.

4. Sapag A, Wouters J, Lambert C, de Ioannes P, Eyzaguirre J, Depiereux E. The endoxylanases from family 11: computer analysis of protein sequences reveals important structural and phylogenetic relationships. J Biotechnol 2002;95:109-131.

5. Törrönen A, Rouvinen J. Structural comparison of two major endo-1, 4-xylanases from Trichoderma reesei. Biochemistry 1995;34:847-856.

6. Törrönen A, Harkki A, Rouvinen J. Three-dimensional structure of endo-1, 4- $\beta$ - xylanase II from Trichoderma reesei: two conformational states in the active site. EMBO J 1994;13:2493-2501.
7. Collins T, Gerda C, Feller G. Xylanases, xylanase families and extremophilic xylanases. FEMS Microbiol Rev 2005;29:3-23.

8. Davies GJ, Wilson KS, Henrissat B. Nomenclature for sugar-binding subsites in glycosyl hydrolases. Biochem J 1997;321:557-559.

9. Turunen O, Vuorio M, Fenel F, Leisola M. Engineering of multiple arginines into the Ser/Thr surface of Trichoderma Reesei endo-1, 4$\beta$-xylanase II increases the thermotolerance and shifts the $\mathrm{pH}$ optimum towards alkaline pH. Protein Eng 2002;15:141-145.

10. Sung WL, Yaguchi M, Ishikawa K. Modification of xylanase to improve thermophilicity, alkalophilicity and thermostability. U.S. Patent 5,759,840, 1988.

11. Sun JY, Liu MQ, Xu YL, Xu ZR, Pan L, Gao H. Improvement of the thermostability and catalytic activity of a mesophilic family 11 xylanase by N-terminus replacement. Protein Expr Purif 2005;42: 122-130.

12. Fenel F, Leisola M, Janis J, Turunen O. A de novo designed N-terminal disulphide bridge stabilizes the Trichoderma reesei endo-1,4- $\beta$ xylanase II. J Biotechnol 2004;108:137-143.

13. Shibuya H, Kaneko S, Hayashi K. Enhancement of the thermostability and hydrolytic activity of xylanase by random gene shuffling. Biochem J 2000;349:651-656.

14. Palackal N, Brennan Y, Callen WN, Dupree P, Frey G, Goubet F, Hazlewood GP, Healey S, Kang YE, Kretz KA, Lee E, Tan X, Tomlinson GL, Verruto J, Wong VW, Mathur EJ, Short JM, Robertson $\mathrm{DE}$, Steer BA. An evolutionary route to xylanase process fitness. Protein Sci 2004;13:494-503.

15. Flatman R, McLauchlan WR, Juge N, Furniss C, Berrin JG, Hughes RK, Manzanares P, Ladbury JE, O’Brien R, Williamson G. Interactions defining the specificity between fungal xylanases and the xylanase-inhibiting protein XIP-I from wheat. Biochem J 2002;365:773-781.

16. Juge N, Payan F, Williamson G. XIP-I, a xylanase inhibitor protein from wheat: a novel protein function. Biochem Biophys Acta 2004; 1696:203-211.

17. Juge N, Delcour JA. Xylanase inhibitors: structure, function and evolution. Curr Enzyme Inhib 2006;2:29-35.

18. Kongsted J, Ryde U, Wydra J, Jensen JH. Prediction and rationalization of the $\mathrm{pH}$ dependence of the activity and stability of family 11 xylanases. Biochemistry 2007;46:13581-13592.

19. Joshi MD, Sidhu G, Pot I, Brayer GD, Withers SG, McIntosh LP. Hydrogen bonding and catalysis: a novel explanation for how a single amino acid substitution can change the $\mathrm{pH}$ optimum of a glycosidase. J Mol Biol 2000;299:255-279.

20. Fushinobu S, Ito K, Konno M, Wakagi T, Matsuazawa H. Crystallographic and mutational analyses of an extreme acidophilic and acid-stable xylanase: biased distribution of acidic residues and importance of Asp 37 for catalysis at low pH. Protein Eng 1998; 11:1121-1128.

21. De Lemos Esteves F, Ruelle V, Lamotte-Brasseur J, Quinting B, Frere JM. Acidophilic adaptation of family 11 endo-beta-1,4-xylanases: modelling and mutational analysis. Protein Sci 2004;13:1209-1218.

22. Berrin JG, Ajandouz EH, Georis J, Arnaut F, Juge N. Substrate and product hydrolysis specificity in family 11 glycoside hydrolases: an anlysis of Penicillium funiculosum and Penicillium griseofulvum xylanases. Appl Microbiol Biotechnol 2007;74:1001-1010.

23. Payan F, Leone P, Porciero S, Furniss C, Tahir T, Williamson G, Durand A, Manzanares P, Gilbert HJ, Juge N, Roussel A. The dual nature of the wheat xylanase protein inhibitor XIP-I. J Biol Chem 2004;279:36029-36037.

24. Emanuelsson O, Nielsen H, Brunak S, von Heijne G. Predicting subcellular localization of proteins based on their N-terminal amino acid sequence. J Mol Biol 2000;300:1005-1016.

25. Nielsen H, Engelbrecht J, Brunak S, von Heijne G. Identification of prokaryotic and eukaryotic signal peptides and prediction of their cleavage sites. Protein Eng 1997;10:1-6.

26. Altschul SF, Madden TL, Schaffer AA, Zhang J, Zhang Z, Miller W, Lipman DJ. Gapped BLAST and PSI-BLAST: a new generation of protein database search programs. Nucleic Acids Res 1997;25:3389-3402. 
27. Jonniaux JL, Dauvrin T. Enzyme with xylanase activity. EP Patent 1,130,102-A1, 2001.

28. Thompson JD, Higgins DG, Gibson TJ. CLUSTAL W: improving the sensitivity of progressive multiple sequence alignment through sequence weighting, position-specific gap penalties and weight matrix choice. Nucleic Acids Res 1994;11:4673-4680.

29. Notredame C, Higgins D, Heringa J. T-Coffee: a novel method for multiple sequence alignments. J Mol Biol 2000;302:205-217.

30. Gouet P, Courcelle E, Stuart DI, Metoz F. ESPript: multiple sequence alignments in PostScript. Bioinformatics 1999;15:305-308.

31. Salí A, Blundell TL. Comparative protein modelling by satisfaction of spacial restraints. J Mol Biol 1993;234:779-815.

32. Laskowski RA, MacArthur MW, Moss DS, Thornton JM. PROCHECK: a program to check the stereochemical quality of protein structures. J Appl Cryst 1993;26:283-291.

33. André-Leroux G, Tessier D, Bonnin E. Action pattern of Fusarium moniliforme endopolygalacturonase towards pectin fragments: comprehension and prediction. Biochim Biophys Acta 2005;1749:53-64.

34. Holm L, Park J. DaliLite workbench for protein structure comparison. Bioinformatics 2000;16:566-567.

35. Notenboom V, Boraston AB, Williams SJ, Kilburn DG, Rose DR. High-resolution crystal structures of the lectin-like xylan binding domain from Streptomyces lividans xylanase 10A with bound substrates reveal a novel mode of xylan binding. Biochemistry 2002; 41:4246-4254.

36. Sabini E, Wilson KS, Danielsen S, Schulein M, Davies GJ. Oligosaccharide binding to family 11 xylanases: both covalent intermediate and mutant product complexes display $(2,5) \mathrm{B}$ conformations at the active center. Acta Crystallogr D Biol Crystallogr 2001;57:13441347.

37. Paès G.Etude structure/fonction d'hémicellulases thermostables: la xylanase GH-11 et l'arabinofuranosidase GH-51 de Thermobacillus xylanilyticus. PhD Thesis, Université de Champagne Ardenne, France, 2005.

38. McLauchlan WR, Garcia-Conesa MT, Williamson G, Roza M, Ravestein P, Maat J. A novel class of protein from wheat which inhibits xylanases. Biochem J 1999;338:441-446.

39. Sidhu G, Withers SG, Nguyen NT, McIntosh LP, Ziser L, Brayer GD. Sugar ring distortion in the glycosyl-enzyme intermediate of a family GH11 xylanase. Biochemistry 1999;8:5346-5354.
40. Wakarchuk WW, Campbell RL, Sung WL, Davoodi J, Yaguchi M. Mutational and crystallographic analyses of the active site residues of the Bacillus circulans xylanase. Protein Sci 1994;3:467-475.

41. Vyas NK. Atomic features of protein-carbohydrates interactions. Curr Opin Struct Biol 1991;1:732-740.

42. Biely P, Vrsanka M, Tenkanen M, Kluepfel DJ. Endo-beta-1,4-xylanase families: differences in catalytic properties. J Biotechnol 1997; 57:151-166.

43. Wouters J, Georis J, Engher D, Vandenhaute J, Dusart J, Frere JM, Depiereux E, Charlier P. Crystallographic analysis of family 11 endo-beta-1,4-xylanase Xyl1 from Streptomyces sp. S38 2001. Acta Crystallogr D Biol Crystallogr 2001;57:1813-1819.

44. Krengel U, Dijkstra BW. Three dimensional structure of endo-1, 4$\beta$-xylanase I from Aspergillus niger: molecular basis for its low $\mathrm{pH}$ optimum. J Mol Biol 1996;263:70-78.

45. Cremer D, Pople JA. A general definition of ring puckering coordinates. J Am Chem Soc 1975;97:1354-1358.

46. Joshi MD, Sidhu G, Nielsen JE, Brayer GD, Withers SG, McIntosh LP. Dissecting the electrostatic interactions and $\mathrm{pH}$-dependant activity of a family 11 glycosidase. Biochemistry 2001;40:10115-10139.

47. Tahir TA, Berrin JG, Flatman R, Roussel A, Roepstorff P, Williamson G, Juge N. Specific characterization of substrate and inhibitor binding sites of a glycosyl hydrolase family 11 xylanase from Aspergillus niger. J Biol Chem 2002;277:44035-44043.

48. Belien T, Van Campenhout S, Van Acker M, Robben J, Courtin CM, Delcour JA, Volckaert G. Mutational analysis of endoxylanases XylA and XylB from the phytopathogen Fusarium graminearum reveals comprehensive insights into their inhibitor insensitivity. Appl Environ Microbiol 2007;73:4602-4608.

49. Belien T, Van Campenhout S, Bosch AV, Bourgois TM, Rombouts S, Robben J, Courtin CM, Delcour JA, Volckaert G. Engineering molecular recognition of endoxylanase enzymes and their inhibitors through phage display. J Mol Recognit 2007;20:103-112.

50. Cortès J, Siméon T, Ruiz de Angulo V, Guiyesse D, Remaud-Siméon M, Tran V. A path planning approach for computing large-amplitude motion of flexible molecules. Bioinformatics 2005;21:116125.

51. Miyazaki T, Takenouchi M, Kondo H, Noro W, Suzuki M, Tsuda S. Thermal stabilization of Bacillus subtilis family 11 xylanase by directed evolution. J Biol Chem 2006;281:10236-10242. 